\title{
Surface engineering of titanium by multi-interstitial diffusion using plasma processing
}

\author{
M. Drouet ${ }^{1}$; L. Pichon ${ }^{1}$, J.B. Dubois ${ }^{1}$, E. Le Bourhis ${ }^{1}$, T. L. Christiansen ${ }^{2}$ \\ 1-Institut Pprime, UPR3346, CNRS Université de Poitiers ISAE-ENSMA, Département Physique et Mécanique des Matériaux, SP2MI TSA 41123, \\ 2-Technical University of Denmark, Department of Mechanical Engineering, Produktionstorvet b.425, DK
}

2800 Kgs. Lyngby, Denmark

\section{Abstract}

Titanium and its alloys possess a range of highly interesting properties such as excellent corrosion resistance, high specific strength and biocompatibility, but suffers from poor wear resistance. The present work addresses plasma assisted surface treatment of CP 2 titanium using various combinations of oxygen and nitrogen, i.e. mixed interstitials. The sequence of controlled plasma nitriding and oxidizing treatments plays a significant role for the evolution of the hardness depth profiles and the development of the surface compound layer and the underlying diffusion/transition zone. Composition profiles of oxygen and nitrogen are obtained by GDOES; Mixed interstitial solubility of nitrogen and oxygen is found in both h.c.p. $\alpha$ titanium and in the compound layer. The combination of interstitials leads to larger case depth, in particular for the diffusion zone (expanded h.c.p. $\alpha$ titanium). Therefore, it highlights the advantages of combined nitriding and oxidizing compared to single nitriding treatments on the mechanical properties.

\section{Introduction}

Titanium alloys are widely used when high specific strength and light weight are required properties like in aerospace industry for example. They also present high corrosion performance, superior to most of iron-based materials, that make them very interesting for chemical industry. These very high corrosion performances result from their ability to spontaneously form a strongly adherent and self-passivating oxide layer. Finally, due to their biocompatibility, they are an ideal choice for biomedical applications like implants. Moreover, the use of titanium-based materials instead of stainless steel avoids the release of toxic nickel in the body. Despite these interesting properties, titanium alloys suffer from poor tribological properties that limit their use in applications under wear solicitations. Surface thermochemical treatments have proved to be efficient ways to remedy this detrimental behaviour inherent to titanium and its alloys. In this paper, we explore new routes to surface hardening based on sequential plasma-enhanced thermo-diffusion of nitrogen and oxygen.

\section{$\underline{1-\text { Context }}$} Due to the strong affinity of titanium with oxygen and the propensity to form oxides, surface hardening of titanium via conventional thermo-chemical processes involving the interstitial elements
carbon and/or nitrogen requires relatively high temperatures and high purity gas systems. It is difficult to avoid formation of oxides which can act as a barrier for other interstitial elements carbon and/or nitrogen requires relatively high temperatures and high purity gas systems. It is difficult to avoid formation of oxides which can act as a barrier for other interstitial elements
incorporation. In gaseous nitriding for example, titanium is exposed to high purity nitrogen containing gas at, say, $1000^{\circ} \mathrm{C}$ to overcome the oxide surface barrier and to achieve significant case depth [1-4]. Another possible method to overcome the oxide barrier is to use plasma enhanced and/or high energy nitriding techniques [5-7]. These techniques enable lower temperatures but at the cost of a relatively thinner diffusion layer, as a consequence of slower diffusion. Therefore, this results in the formation of a surface zone constituted of very hard layers of TiN and $\mathrm{Ti}_{2} \mathrm{~N}$ compounds, and a supporting diffusion zone of variable thickness [8,9]. These nitride layers have high hardness and low friction coefficient.

Contrary to nitriding, limited work is available in literature concerning carbon incorporation. Despite the high temperature used in a carburizing process, the very low solubility of carbon in titanium results in essentially no diffusion zone. Therefore, these treatments result in an extremely hard and thin $\mathrm{Ti}_{1-\mathrm{x}} \mathrm{C}$ carbide layer on a relatively soft substrate, which is unwanted due to the risk of "egg shell" effect [10-12]

High temperature oxidation is usually considered a corrosion mechanism which is detrimental for the materials properties. However, in the case of titanium, due to the very high solubility of oxygen (up to $32.4 \mathrm{at} \%$ ) and the strong interstitial strengthening effect, oxygen can - perhaps counterintuitively - be used for surface hardening. Surface hardening of titanium with oxygen has already been investigated in the 1960's, although not as extensively as nitriding process. Oxidizing takes advantage of the high solubility of oxygen in titanium to form relatively thick diffusion zone [13-16].

To summarize, the three interstitial elements have different but somehow complementary diffusion behaviours. It appears then promising to combine them in the form of a mixed interstitial compound or as solid solutions in titanium. According to Fedirko [17], the ternary compounds have higher hardness and wear resistance than any of the binary compounds. Unfortunately, all these high temperature processes can significantly impact the microstructure and properties of the core material [18]. In order to circumvent unwanted deterioration of bulk properties, lower temperature processes have to be applied. In particular, unaltered mechanical properties are of importance in, for example, aeronautics.

In this paper, we present a first step on the route to "low" temperature surface hardening of titanium using ternary mixed-interstitial by the use of plasma-enhanced processes. The goal is to obtain high wear resistance all while preserving core properties.

\section{$\underline{\text { 2- Experiments }}$}

Commercially pure grade 2 titanium was used for this study. Grade 2 titanium has an h.c.p. a structure at room temperature and was chosen as a reference material as it represents the pure titanium system without major allying element, i.e. the simplest possible microstructure. The grain size in the material was several tens of microns. $4 \mathrm{~mm}$ thick and $30 \mathrm{~mm}$ diameter samples were cut out of the received bar. They were then polished using the 3 steps Struers® protocol with colloidal silica as the final step. A similar protocol, but with additional intermediary steps in order to minimise work hardening, was used to prepare cross sections for Scanning Electron Microscopy (SEM) and micro-hardness measurements. The thermo-chemical treatments were conducted in a custom built low-pressure plasma assisted reactor [19]. Briefly, this reactor consists in a quartz tube fitted with an external Radio Frequency (RF) electrode and surrounded by a furnace. The samples are introduced in the reactor and kept in the "cold" zone during the pre-heating of the furnace to the treatment temperature. For each treatment step, an appropriate gas mixture containing the active gas is introduced in the tube and the pressure is set at the desired value. When necessary, RF power, is injected in the system to light up the plasma. The sample is then transferred in the working temperature zone of the rig. The sample heats up to the process temperature in approximately 10 minutes, the duration of this step can be neglected compared to the total process time of several hours. At the end of the treatment cycle, the sample is brought back in the cold zone and rapidly cooled down to $350{ }^{\circ} \mathrm{C}$ (within about 10 min). This temperature is low enough to prevent any significant additional diffusion.

The chemical composition of the obtained surface compounds and diffusion layers was measured by Glow Discharge Optical Emission Spectroscopy (GDOES) and confirmed by Energy Dispersive $\mathrm{X}$-ray spectroscopy (EDX) on cross sections. The phases were identified from X-Ray Diffraction (XRD) patterns in Q-2Q geometry with $\mathrm{Cu}_{\mathrm{K} \alpha}$ radiation.

The hardness profiles were obtained with a protocol adopted to ensure both a significant number of data points across the treated zone of the sample and no influence of the points on each other. The tests were performed with a load of $0.245 \mathrm{~N}$ and a dwell time of $10 \mathrm{~s}$. Lines of $15-20$ indents separated by $50 \mu \mathrm{m}$ were made along a direction inclined by $80-85^{\circ}$ to the surface normal, resulting in an increment in distance from the surface of approximately 5-8 $\mu \mathrm{m}$. The hardness was determined as the mean pressure over the contact surface calculated from the two diagonal sizes of Vickers mark.

Following the idea of bulk microstructure preservation, 2 treatment temperatures, well below the b transus of grade 2 titanium $\left(913^{\circ} \mathrm{C}\right)$, were tested: $750{ }^{\circ} \mathrm{C}$ and $850{ }^{\circ} \mathrm{C}$. In a first step, simple nitriding was applied at both temperatures using our standard plasma conditions, $60 \% \mathrm{~N}_{2}-40 \% \mathrm{H}_{2}$ at $50 \mu$ bar and $700 \mathrm{~W}$ RF power that have been proved to be a very efficient nitriding medium, as

(C) The Authors, published by EDP Sciences. This is an open access article distributed under the terms of the Creative Commons Attribution License 4.0 (http://creativecommons.org/licenses/by/4.0/). 
a reference "state of the art" process $[5,20,21]$. Combined treatments using sequential nitriding, oxidizing and vacuum diffusion, at residual pressure of $10^{-6}$ mbar, were then tested. Oxidizing sequences were performed with $50 \mu$ bar of pure oxygen and $700 \mathrm{~W}$ RF power was injected for plasma oxidizing. The different treatment cycles are summarized in Table 1 below.

Table 1: Surface treatments sequences used $(\mathrm{PN}=$ plasma nitriding; $\mathrm{VD}=$ vacuum diffusion; $\mathrm{PO}=$ plasma oxidizing; $\mathrm{TO}=$ thermal oxidizing

\begin{tabular}{|l|l|l|l|l|}
\hline Sample A & PN $750^{\circ} \mathrm{C} / 72 \mathrm{~h}$ & & & \\
\hline Sample B & PN $850^{\circ} \mathrm{C} / 8 \mathrm{~h}$ & & & \\
\hline Sample C & PN $850^{\circ} \mathrm{C} / 32 \mathrm{~h}$ & & & \\
\hline Sample D & PN $850^{\circ} \mathrm{C} / 8 \mathrm{~h}$ & VD $850^{\circ} \mathrm{C} / 67 \mathrm{~h}$ & & \\
\hline Sample E & PN $750^{\circ} \mathrm{C} / 72 \mathrm{~h}$ & PO $750^{\circ} \mathrm{C} / 4 \mathrm{~h}$ & & \\
\hline Sample F & PN $850^{\circ} \mathrm{C} / 8 \mathrm{~h}$ & VD $850^{\circ} \mathrm{C} / 15 \mathrm{~h}$ & PO $850^{\circ} \mathrm{C} / 4 \mathrm{~h}$ & \\
\hline Sample G & TO $750^{\circ} \mathrm{C} / 4 \mathrm{~h}$ & VD $850^{\circ} \mathrm{C} / 15 \mathrm{~h}$ & PN $850^{\circ} \mathrm{C} / 8 \mathrm{~h}$ & \\
\hline Sample H & PN $850^{\circ} \mathrm{C} / 8 \mathrm{~h}$ & VD $850^{\circ} \mathrm{C} / 15 \mathrm{~h}$ & TO $750^{\circ} \mathrm{C} / 4 \mathrm{~h}$ & VD $850^{\circ} \mathrm{C} / 15 \mathrm{~h}$ \\
\hline
\end{tabular}

\section{$\underline{3-\text { Results }}$}

3.1 Nitriding

Grade 2 samples were nitrided at $750{ }^{\circ} \mathrm{C}$ for $72 \mathrm{~h}$ and $850^{\circ} \mathrm{C}$ for various durations (see Table 1). The durations of treatments were chosen based on diffusion coefficients determined by Anttila et al [22] in order to obtain a technologically usable case depth. For all tested conditions, the nitriding treatment leads to a thin compound layer (a few $\mu \mathrm{m}$ ) which was found to mainly consist of $\mathrm{Ti}{ }_{2} \mathrm{~N}$ as deduced from GDOES composition analysis (Fig. 1) and X-Ray diffraction patterns (Fig. 2a). Underneath this compound layer, a diffusion tail is present. Although too thin to be correctly detected on GDOES profile, a golden coloured TiN layer is also present at the outermost surface. As expected, nitriding is more effective at higher temperature, considering the compound layer that is thicker as a direct consequence of enhanced solubility and increased diffusion coefficient. The nitrogen concentration in the diffusion tail is higher and extends deeper for longer cycles (samples $\mathrm{C}$ and $\mathrm{D}$ ). Using a $1 \%$ concentration criteria, the diffusion length fits well the square-root dependence with time, associated to a reduction of the nitrogen concentration in the case of sample $\mathrm{D}$ with a vacuum diffusion sequence. However, the total case depth remains modest.

Fig. 3 shows the cross section of a sample nitrided for 8 hours at $850{ }^{\circ} \mathrm{C}$ (sample B) together with the corresponding GDOES profile. The microstructure of the Ti ${ }_{2} \mathrm{~N}$ surface layer is composed of small (compared to the initial microstructure) grains elongated in the growth direction. This is consistent with the textured structure that can be deduced from the X-Ray diffraction pattern (Fig. $2 \mathrm{a}$ ). Below the $\mathrm{Ti}_{2} \mathrm{~N}$ layer, in the diffusion zone (depth range of $4-20 \mu \mathrm{m}$ ), no features can be observed. This is in stark contrast to the bulk microstructure, which contains dark marks of few $\mu \mathrm{m}$, most likely a consequence of surface polishing. The absence of this feature in the nitrogen containing zone suggests a significant hardening of the material

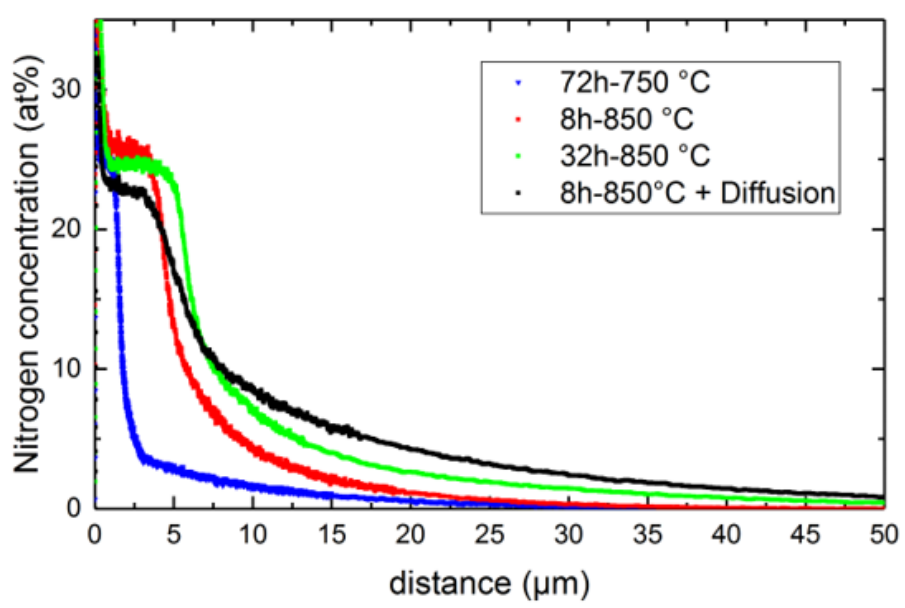

Figure 1: GDOES nitrogen concentration profiles obtained after $8 \mathrm{~h}$ nitriding at $750^{\circ} \mathrm{C} 72 \mathrm{~h}$ (blue), $850^{\circ} \mathrm{C} 8 \mathrm{~h}$ (red), $850^{\circ} \mathrm{C} 32 \mathrm{~h}$ (green) and $850^{\circ} \mathrm{C}$ followed by $67 \mathrm{~h}$ vacuum diffusion (black) corresponding to samples A, $\mathrm{B}, \mathrm{C}$ and D respectively. 

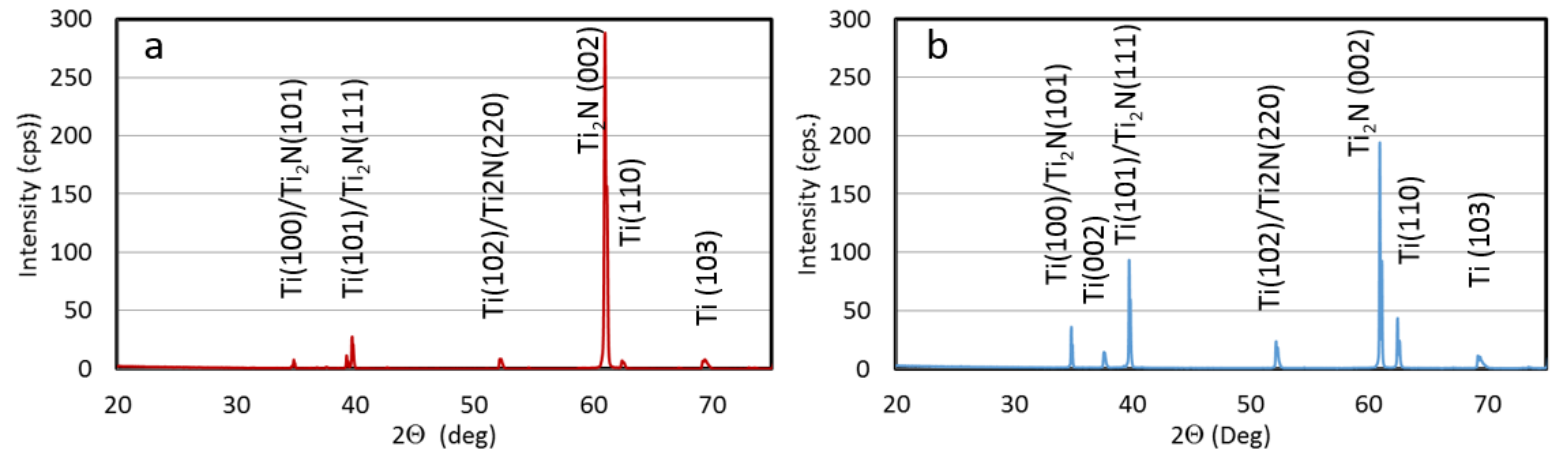

Figure 2: (a) X-Ray diffraction patterns obtained after $8 \mathrm{~h}$ nitriding + diffusion (Sample D) and (b) optimised $8 \mathrm{~h}$ nitriding +oxidizing treatment sequences (Sample $\mathrm{H}$ ). The main peak corresponds to Ti $2 \mathrm{~N}$ ( 002 ), all others have contributions of $\mathrm{Ti}_{2} \mathrm{~N}$ and Nitrogen/Oxygen saturated a-Ti. Notice the absence of $\mathrm{TiO}_{2}$ peak for the mixt treatment.

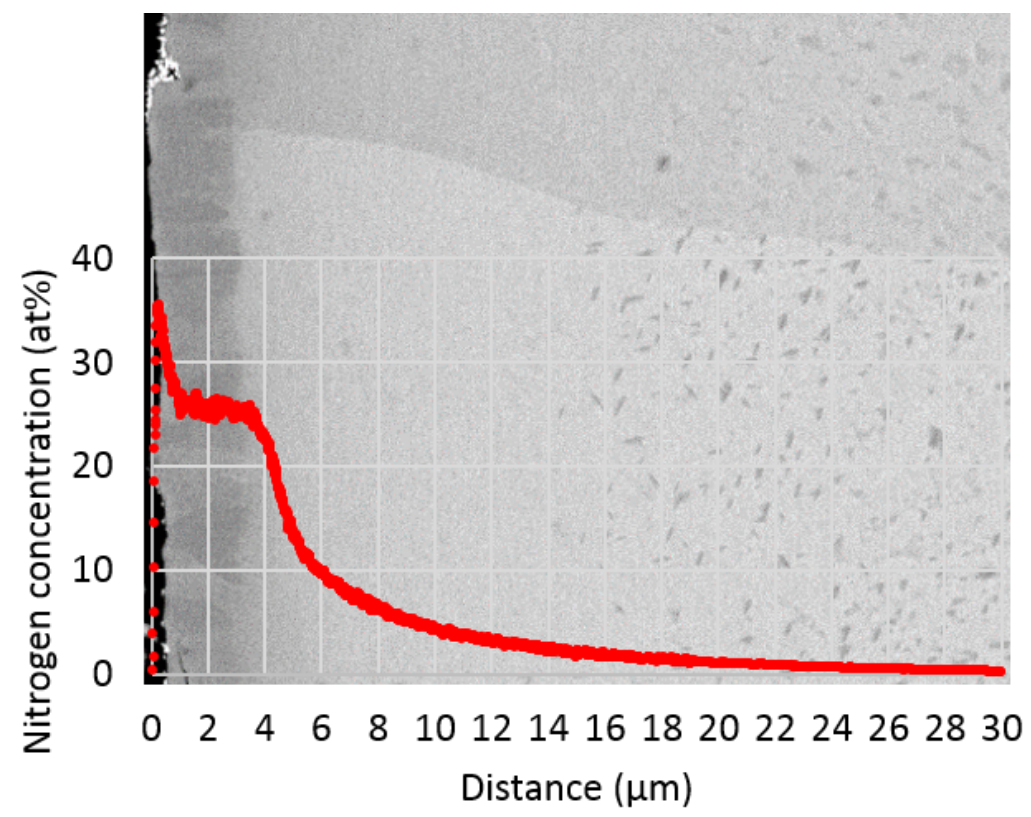

Figure 3: Scanning Electron Micrograph (backscattering electrons composition mode) of cross section of sample nitrided for $8 \mathrm{~h}$ at $850^{\circ} \mathrm{C}$ (sample B). The compound layer, approximately $4 \mu \mathrm{m}$ thick is constituted of small grains elongated normal to the surface. This layer corresponds to the concentration plateau as can be seen on the superimposed GDOES nitrogen concentration profile. Notice the absence of dark marks in the diffusion zone (see text)

3.2 Combined nitriding and oxidizing

Combined nitriding/oxidizing treatments were performed based on the above nitriding sequences. For plasma oxidizing at $750{ }^{\circ} \mathrm{C}$ (sample E), a very thin oxide layer, less than $1 \mu \mathrm{m}$ as measured by GDOES (not presented), is formed but no significant diffusion of oxygen is observed. At $850^{\circ} \mathrm{C}$, a thicker layer, consisting of $\mathrm{TiO}_{2}$ rutile of estimated thickness of $13 \mu \mathrm{m}$, is formed but it spalls off on cooling, leaving behind a large diffusion zone with significant amount of oxygen down to $40 \mu \mathrm{m}$ (Fig. 4). The oxygen profile shows a thin, less than $1 \mu \mathrm{m}$, oxygen rich layer is still present at the outermost surface. It is followed by a lower oxygen concentration in the $\mathrm{Ti}_{2} \mathrm{~N}$ layer before growing up again to 8 at. $\%$ at the beginning of the diffusion tail. Interestingly, the spalled-out oxide grew by titanium diffusion toward the surface through the nitride as the $\mathrm{Ti}_{2} \mathrm{~N}$ layer thickness remains mostly the same as for nitriding only (sample $\mathrm{B}$ ). 


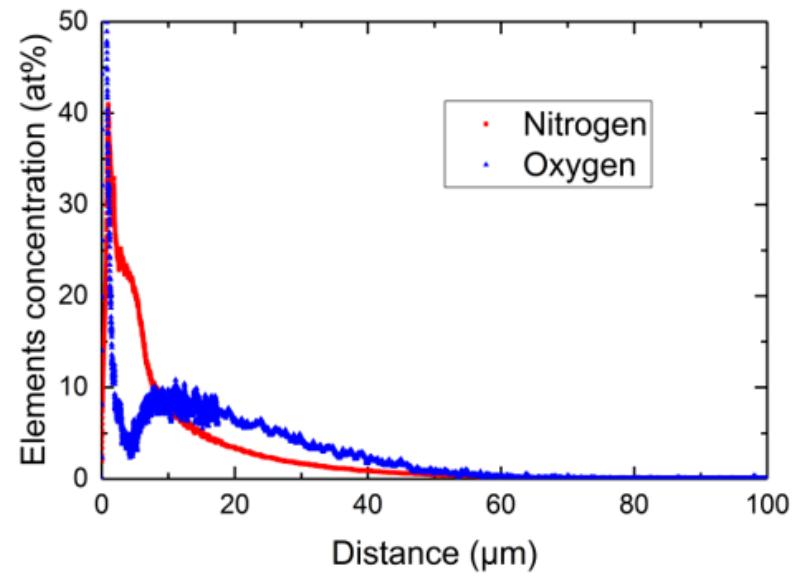

Figure 4: GDOES nitrogen and oxygen profiles in Sample $\mathrm{F}$ ( $8 \mathrm{~h}$ plasma nitriding + diffusion followed by $4 \mathrm{~h}$ plasma oxidizing at $850^{\circ} \mathrm{C}$ ), after the delamination of a $13 \mu \mathrm{m}$ thick oxide layer. A very thin oxygen rich layer is still present at the outermost surface as well as an oxygen diffusion profile behind the nitrogen plateau.

Due to the size of the indents and the induced crack when the indent is too close to the surface, micro hardness profiles cannot be obtained in the thin compound layer. However, they give valuable information on the supporting layer (diffusion zone) that plays an essential role in wear resistance. As we can infer from GDOES and SEM observations, nitrided samples (A and B) exhibit similar hardness behaviour (Fig 5) with a decrease of the hardness from $6 \mathrm{GPa}$ close to compound layer to the bulk value $\left(2 \mathrm{GPa}\right.$ ) over $20 \mu \mathrm{m}$. While post oxidizing at $750{ }^{\circ} \mathrm{C}$ (sample $\mathrm{E}$ ) has no measurable influence, a significant improvement is obtained with $850{ }^{\circ} \mathrm{C}$ oxidizing (sample F), leading to both an increase of maximum hardness to $10 \mathrm{GPa}$ and an extension of the affected zone to $90 \mu \mathrm{m}$. Notice that from the hardness point of view, combined nitriding/oxidizing treatment (sample F, red squares) with a $27 \mathrm{~h}$ total duration is more efficient than single nitriding plus diffusion (sample D, green stars) which is 3 time longer.

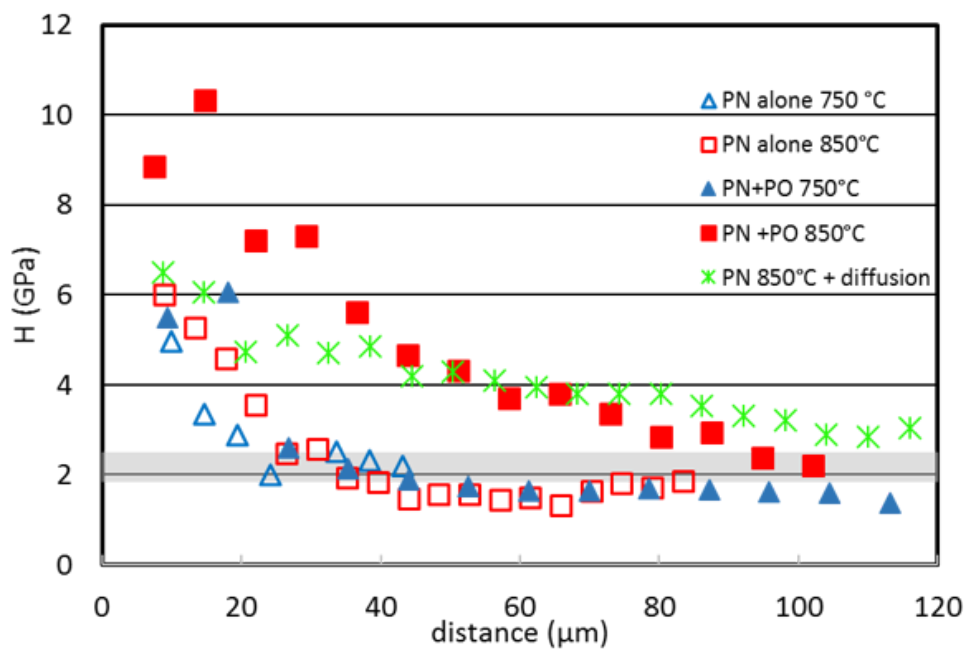

Figure 5: Effect of post oxidizing on the micro hardness profile for the 2 studied temperatures, $750{ }^{\circ} \mathrm{C}$ (blue triangles, sample E) and $850{ }^{\circ} \mathrm{C}$ (red squares, sample F). Single nitriding + diffusion profile (green stars, sample D) is plotted for comparison. Grey band corresponds to the substrate hardness before treatment.

In order to improve the efficiency of the treatments, e.g. to avoid oxide layer delamination, different nitriding, oxidizing and diffusion sequences were tested: realizing the oxidizing sequence prior to nitriding, lowering the oxidizing temperature to $750^{\circ} \mathrm{C}$ and switching to pure thermal oxidizing at low $\mathrm{O}_{2}$ pressure $(50 \mu$ bar). Figure 6 presents the results for two combined cycles of plasma nitriding thermal oxidizing and diffusion. Thermal oxidizing took place before nitriding for sample $\mathrm{G}$ and after nitriding for sample $\mathrm{H}$. No delamination of any compound layer was observed after both treatments. In both cases the oxygen concentration is low, except at the outermost surface but the nitrogen profiles are quite different. If the nitrogen profile of Fig. $6 \mathrm{a}$ is compared to the one obtained for the same nitriding conditions (sample B in Fig. 1), it is clear that the compound layer is absent and the penetration depth is reduced. This can be attributed to a lower incorporation rate due to the surface oxide layer. In Fig. $6 \mathrm{~b}$, where thermal oxidizing $\left(750^{\circ} \mathrm{C}\right) /$ diffusion $\left(850^{\circ} \mathrm{C}\right)$ was done after $8 \mathrm{~h}$ plasma nitriding and $15 \mathrm{~h}$ vacuum diffusion at $850^{\circ} \mathrm{C}($ sample $\mathrm{H})$, the surface nitride has been partially transformed in oxide and the nitrogen diffusion length is increased due to the additional diffusion time. The XRD pattern of this sample is shown in Fig. 2b: similar to nitrided sample (Fig. 2a), the $\mathrm{Ti}_{2} \mathrm{~N}(002)$ peak is the prominent one, at the same position. However, no oxide peak can be observed. In these last two combined treatments, the micro-hardness profiles show a significant enhancement, as compared to the one obtained with a single nitriding step of similar duration (Fig. 7 black triangles, sample C). The profile are actually almost equivalents to the one obtained for a long post nitriding vacuum diffusion (green stars, sample D) both in terms of maximum hardness (6-7 GPa) and extension $(\approx 100 \mu \mathrm{m})$. Their behaviour are however different in details as sample $\mathrm{G}$ exhibits a hardness plateau at $4 \mathrm{GPa}$ between 20 and $60 \mu \mathrm{m}$ while sample $\mathrm{H}$ hardness decreases linearly. 

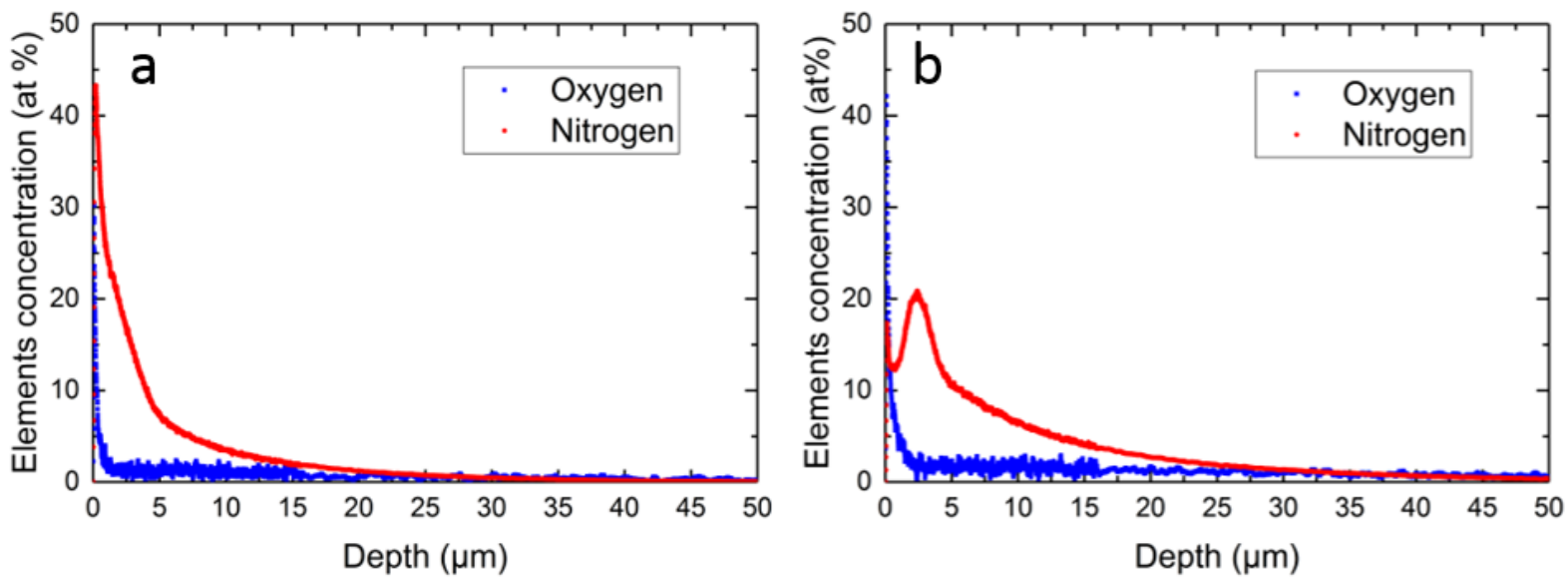

Figure 6: Oxygen and nitrogen profiles after 2 different combined cycles. (a)-oxidizing/diffusion before nitriding (Sample G) and (b)-oxidizing/diffusion after nitriding (Sample H).

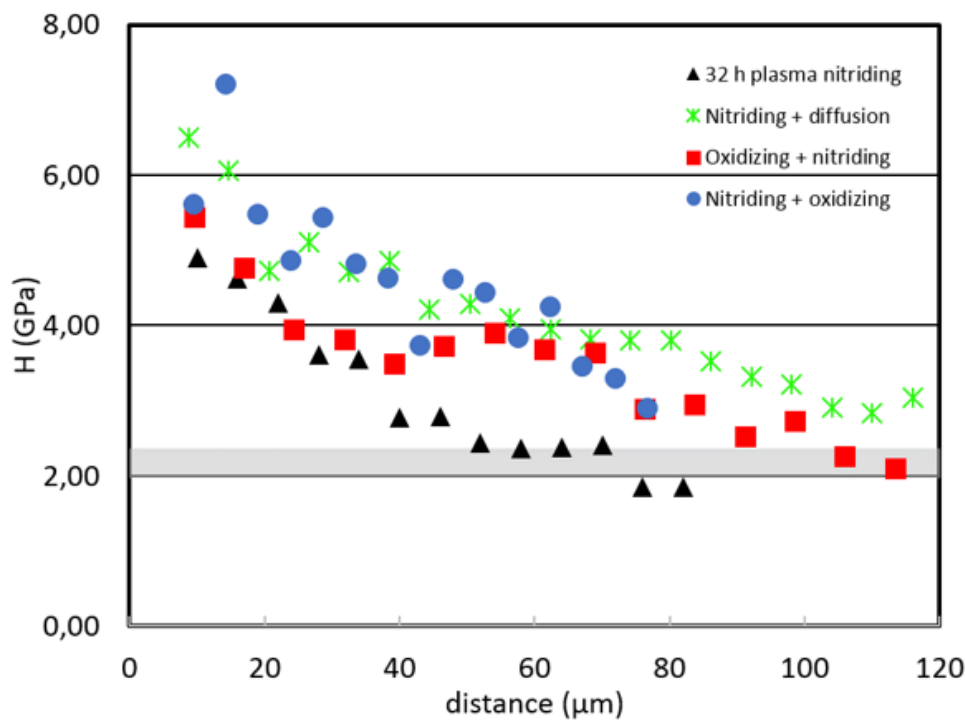

Figure 7: Effect of treatments on the microhardness of the supporting layer for different treatment conditions: single 32h long plasma nitriding (black triangles, Sample C), nitriding + diffusion (green stars, sample D), oxidizing + nitriding (red squares, Sample G) and nitriding + oxidizing (blue dots, Sample H). Grey band corresponds to the substrate hardness before treatment.

$\underline{\text { Conclusion }}$

Surface hardening of titanium was achieved at moderate temperatures using plasma enhanced nitriding and oxidizing. For all investigated conditions, surface layers of nitrides and oxides of various thicknesses were formed. However, to confer wear resistance to the material a smooth hardness transition from the compound layer to the substrate is required. At the lowest investigated temperature $\left(750^{\circ} \mathrm{C}\right)$, this transition is quite abrupt (e.g. over a distance comparable to the layer thickness) and will not fulfil this requirement. The optimal condition was found with a combination of $750{ }^{\circ} \mathrm{C} /$ $850{ }^{\circ} \mathrm{C}$ treatments, where the thickness of the transition/diffusion zone is one order of magnitude larger than the compound layer and without delamination of any compound layer. The temperature $850{ }^{\circ} \mathrm{C}$ is considered a suitable temperature for both preservation of the bulk mechanical properties, as measured by micro hardness, and efficient surface hardening. It is clear that combination of 2 interstitial elements, nitrogen and oxygen, greatly enhances the efficiency of the treatments compared to single step nitriding. This work is a first step in plasma assisted tailoring of the surface properties of titanium based on mixed interstitial phases. Further improvement is expected from the use of carbon as third interstitial element.

Acknowledgments: This work was partially funded by Danish DFF-Research Project 2 MixTi. The authors thank K. Drouet, master student in translation in an intercultural context at the FTI/EII UMons 7000 Mons, Belgium, for English proof reading.

\section{References:}

[1] A. Zhechevaa, W. Shaa, S. Malinovb, A. Longa; Surface \& Coatings Technology 200 (2005) $2192-2207$

[2] A. Zhecheva, S. Malinov, I. Katzarov and W. Sha; Surface Engineering 2006 VOL 22 NO 6: 452

[3] A.E. Barington, M.S. Jellesen, T.L. Christiansen, 29th International Conference on Surface Modification Technologies, 10-12 June 2015, Copenhagen, Denmark, proceedings. 
[4] T.L. Christiansen, M.S. Jellesen, M.A.J. Somers, Metallurgia Italiana, 9 (2018) 13-22

[5] V. Fouquet, L. Pichon, M. Drouet, A. Straboni, Applied Surface Science, Volume 221, Issues 1-4 ( 2004) 248-258

[6] V. Fouquet, L. Pichon, A. Straboni and M. Drouet, Surf. Coating Technol 186, (2004) 34-39

[7] V.M.C.A. Oliveiraa, M.O.H. Cioffia, M.J.R. Barbozab, R. Landersc, B. Schmittd, D.C.A.R. Tapiae,H.J.C. Voorwalda; International Journal of Fatigue 109 (2018) $157-165$

[8] V. Fouquet, Le Bourhis, L. Pichon, M. Drouet and A.Straboni, Scripta Materialia, Volume 51, Issue 9, (2004) Pages 899-903

[9] V.N. Fedirko, T.N. Pogrelyuk, Soviet Materials Science 19(6) (1984) 511-513

[10] Y. Luo, S. Ge, D. Zhang, Q. Wang, H. Liu, Tribology International 44 (2011) 1471-1475

[11] I.V. Nikonorova, E.L. Gyulikhandanov, Metal Science and Heat Treatment 40(1-2) (1998) 52-53.

[12] P.K. Ajikumar, M. Vijayakumar, M. Kamruddin, S. Kalavathi, N. Kumar, T.R. Ravindran, A.K. Tyagi; Int. Journal of Refractory Metals and Hard Materials 31 (2012) 62-70 [13] C Hertl, E Werner, R Thull and U Gbureck; Biomed. Mater. 5 (2010) 054104

[14] H. Dong, X.Y. Li; Materials Science and Engineering A280 (2000) $303-310$

[15] A. Takamura, Trans. "Surface Hardening of Titanium by Oxygen”, JIM 3 (1962) 10-14.

[16] Y.Z. Kim, T. Konno, T. Murakami, T. Narushima, C. Ouchi, Materials Transactions 50(12) (2009) 2763-2771.

[17] V.M. Fedirko, I.M. Pohrelyuk, O.I. Yas'kiv, Materials Sciences 42(3) (2006) 299-308

[18] N. Gammeltoft-Hansen, S. Munch, M. Jellesen, M. Somers, T. Christiansen; Materials performance and characterization; Volume: 6 Issue: 3 Special Issue: SI Pages: $298-310$ [19] J. Perriere, J. Siejka, N. Remili, A. Laurent, A. Straboni, B. Vuillermoz, J. Appl. Phys. 59 Ž1986. 2752.

[20] L. Marot, L. Pichon, M. Drouet, A. Straboni, Mater. Lett. 44 (2000) 35-38

[21] M. Drouet, J.C.Stinville, P. Villechaise, J.P. Rivière, C. Templier, Eur. Phys. J. Appl. Phys. 43, (2008), 349-351

[22] A. Anttila, J. Raisanen, and J. Keinonen, Appl. Phys. Lett., 42(6) (1983) 498-500 